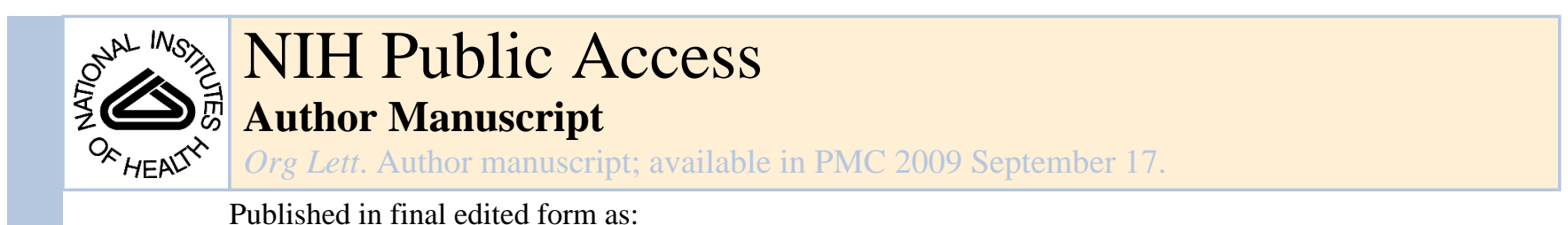

Published in final edited form as:

Org Lett. 2008 April 17; 10(8): 1657-1659. doi:10.1021/ol800395m.

\title{
Efficient, Selective, and Green:
}

\section{Catalyst Tuning for Highly Enantioselective Reactions of Ethylene}

Craig R. Smith and T. V. RajanBabu

Department of Chemistry, The Ohio State University, 100 West 18th Avenue, Columbus, Ohio 43210

\section{Abstract}

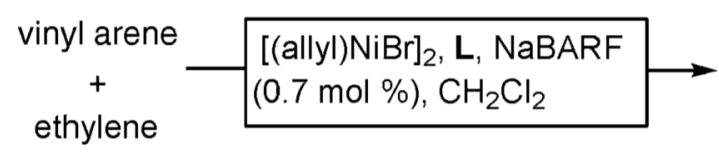<smiles>C=C[C@@H](C)c1ccc(Br)cc1</smiles><smiles>C=C[C@H](C)c1ccc(-c2ccccc2)c(F)c1</smiles>
(yield 97\%; 97\% ee) (yield 99\%; 97\% ee)<smiles>C[C@H](c1cccc2ccccc12)N(Cc1ccccc1)POc1ccc2ccccc2c1-c1c(OP)ccc2ccccc12</smiles><smiles>C=C[C@H](C)c1ccc2cc(OC)ccc2c1</smiles><smiles>C=C[C@]1(C)CCCc2ccccc21</smiles>

(yield 98\%; 99\% ee) (yield 71\%; 99\% ee)

- [4-i-Bu-styrene]/[Ni(II)L] = $7142(0.014 \mathrm{~mol} \%$ cat.) demonstrated - HV of 1-phenyl-1,3-butadiene (up to $84 \%$ ee)

Fine tuning of the biaryl and amino moieties of Feringa's phosphoramidite ligands yields structurally simpler, yet more efficient and selective, ligands for asymmetric hydrovinylation of vinylarenes and acylic 1,3-dienes. The enantioselectivities and yields observed in the formation of the 3-arylbutenes are among the highest for all asymmetric catalytic processes reported to date for the synthesis of intermediates for the widely used antiinflammatory 2 -arylpropionic acids including naproxen, ibuprofen, fenoprofen, and flurbiprofen.

The asymmetric hydrovinylation (HV) of an alkene, viz., addition of ethylene as a vinyl group and a hydrogen across a double bond with concomitant generation of an asymmetric center, is one of the oldest asymmetric carbon-carbon bond-forming reactions. ${ }^{1}$ Since ethylene is a cheap, abundantly available feedstock carbon source, and the resulting vinyl group in the product readily transformed into a variety of other common functional groups, this reaction has huge potential as a scalable, environmentally benign method for the preparation of valuable chemical intermediates.

Recently, several protocols for the reaction have been described in which nearly quantitative yields of the desired products can be obtained using only catalytic amounts of metal complexes, most notably those of nickel (eq 1). ${ }^{2}$ Yet, practical levels of enantioselectivity (i.e., enantiomeric excess $>95 \%$ ) have been achieved only for limited substrates, often at the cost 
of incomplete conversions, isomerization of the primary products, and attendant oligomerization of the starting alkenes.
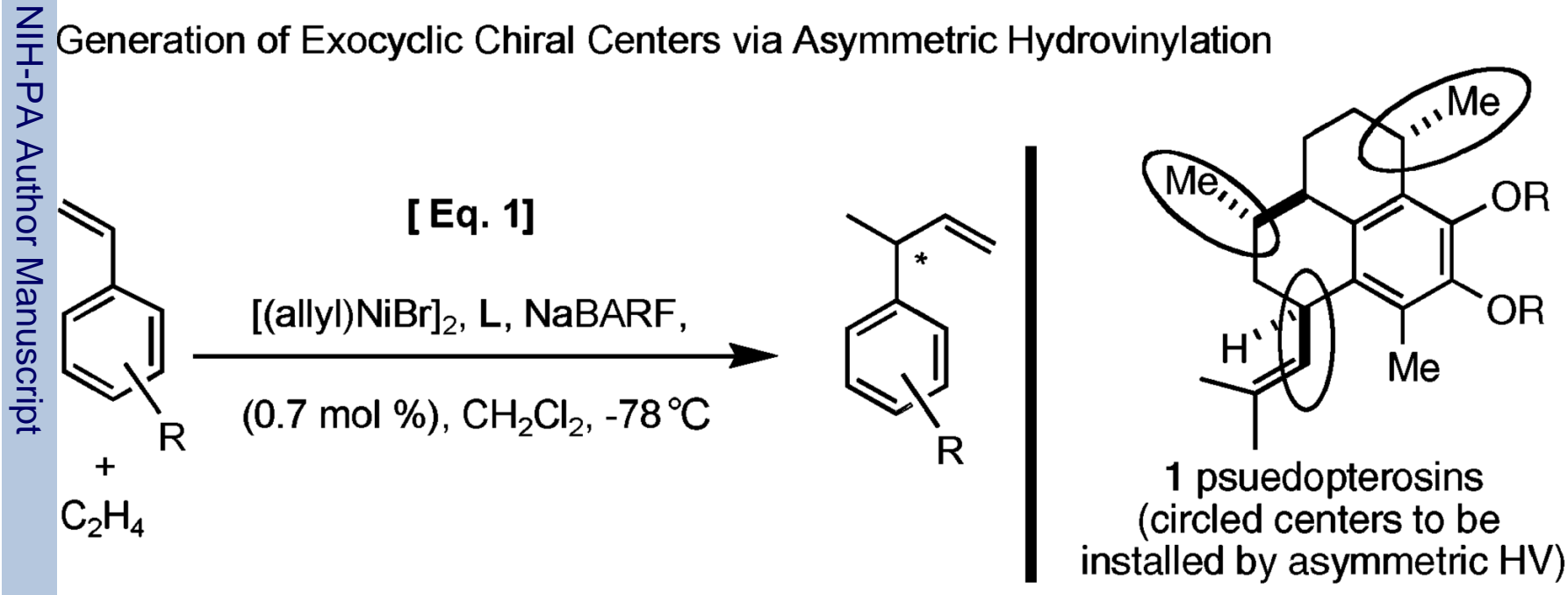

Conspicuously absent among the more successful substrates are Ar-substituted vinylarenes, best exemplified ${ }^{3}$ by 4 -isobutylstyrene (current best: $~ 95 \%$ yield, 91\% ee) and 6-methoxy-2vinylnaphthalene (73\% yield; $86 \%$ ee), potential precursors of antiinflammatory 2arylpropionic acids, $(S)$-ibuprofen and $(S)$-naproxen, the latter a hugely successful commercial drug (Aleve) currently produced by classical resolution. ${ }^{4}$ Since binaphthol-derived phosphoramidites ${ }^{5}$ were introduced for asymmetric HV of vinylarenes, ${ }^{6 a}$ under our originally reported conditions, ${ }^{2 \mathrm{a}}$ these ligands have been used with varying degree of success for $\mathrm{HV}$ of a variety of substrates including norbornene, ${ }^{6 b} 1,3$-dienes, ${ }^{6 c}$ and 1-substituted styrenes. ${ }^{6 \mathrm{~d}, \mathrm{e}}$ Asymmetric HV of similar substrates is the starting point for several total synthesis efforts (e.g., pseudopterosins and related compounds (see eq 1)) in our group; therefore, we decided to explore the scope of ligand tuning in this highly versatile, modular ligand system, and the results are reported in this paper. Ligands $\mathbf{L}_{\mathbf{1}}-\mathbf{L}_{10}$ (Figure 1), readily prepared ${ }^{7,8}$ from the corresponding bisphenols, $\mathrm{PCl}_{3}$, and various chiral amines, were used for this study.

The feasibility of ligand control in the hydrovinylation was initially investigated using $p$ methoxystyrene, an electron-rich model substrate that consistently had given one of the poorest reactions ( $80 \%$ yield, $73 \%$ ee) among vinylarenes tested previously. We started these investigations using a modified protocol (eq $1, \mathrm{R}=4-\mathrm{OMe})$ that had originally been developed for the generation of all-carbon quaternary centers. ${ }^{6 \mathrm{~d}}$ The results are tabulated in Table 1 . A sample of the racemic compound was prepared in a reaction of $p$-methoxystyrene with ethylene $(1 \mathrm{~atm})$ in the presence of a catalytic amount of $[(\text { allyl }) \mathrm{NiBr}]_{2}$, a racemic ligand (a $1: 1$ mixture of $\mathbf{L}_{\mathbf{1}}$ and $\mathbf{L}_{\mathbf{2}}$ ), and sodium tetrakis-[(3,5-trifluoromethyl)phenyl]borate [NaBARF] (Table 1, entry 1 ).

Among the ligands examined, in addition to the original Feringa ligand $\mathbf{L}_{\mathbf{3}}$, two others stand out. $^{8}$ The ligand $\mathbf{L}_{\mathbf{1}}$ (or its enantiomer $\mathbf{L}_{\mathbf{2}}$ ), which has only a lowly biphenyl backbone instead of a chiral binaphthyl unit and is significantly cheaper, still yields similar selectivities and conversions (entries 2 and 3). The ligand $\mathbf{L}_{\mathbf{1 0}}$, in which the $(S)-N$ - $\alpha$-methylbenzyl groups are replaced with achiral benzyl and chiral $(S)$ - $\alpha$-methyl-1-naphthyl groups, is by far the best ligand for this exacting reaction, ${ }^{9}$ yielding nearly quantitative yield and selectivity (entry 17). Surprisingly, ligands prepared from achiral dibenzylamine and enantiopure 2,2'-binaphthol (not shown) gave no conversion. ${ }^{8}$ 
Hydrovinylation of other vinylarenes, 1-alkylvinylarenes, and an open-chain diene was attempted under optimal conditions, and the results are shown in Table 2. The enantioselectivities obtained for the precursors 4-7 for enantiopure arylpropionic acids ibuprofen, naproxen flurbiprofen, and fenoprofen (entries 2-5) represent the highest overall selectivity obtained to date for any viable intermediates for these important compounds. ${ }^{10} \mathrm{In}$ one case where we have further optimized the reaction, the hydrovinylation of 4-i-butylstyrene can be accomplished with 0.00014 equiv of catalyst (substrate/catalyst ratio $=7142$ ) in $4.67 \mathrm{~h}$ at $0{ }^{\circ} \mathrm{C}$. For the biphenyl-derived ligands $\mathbf{L}_{\mathbf{1}}$ and $\mathbf{L}_{\mathbf{2}}$, the configuration of the amine determines the sense of asymmetric induction. With the $S$-chiral moiety in the amine portion of the ligand, the product configuration in all cases is also $S$. As seen in entries 1-5, the lack of axial chirality in the ligand leads to little erosion of ee, suggesting that for simple substrates a more elaborate (and expensive) binaphthol-based phosphoramidite is not necessary to achieve high stereoselectivity. In all cases examined, $\mathbf{L}_{\mathbf{1 0}}$ yielded the best results in terms of overall yield and selectivity.

Although for the vinylarenes, including 1-alkylstyrenes (entries 7 and 8), which yield allcarbon quaternary centers in the product, less rigid catalytic complexes from biphenols are adequate, for more challenging substrates such as an acyclic 1,3-diene, ${ }^{11}$ a binaphthyl backbone is essential for high selectivities (entry 6). Previous attempts to effect asymmetric hydrovinylation of acylic 1,3-dienes resulted in less than 5\% ee. ${ }^{6 c, 12}$ Although 1methylenetetralin undergoes hydrovinylation easily to afford $\mathbf{8}$ in excellent ee, the substrate underwent significant isomerization ( 30\%) of the starting material to 1-methyl-3,4dihydronaphthalene, which is a major distraction from this otherwise useful reaction.

Expansion of the scope of this reaction to heteroaromatic compounds, cyclic vinylarenes, and acyclic dienes and applications of these reactions in natural product synthesis will be reported in due course.

\section{Supplementary Material}

Refer to Web version on PubMed Central for supplementary material.

\section{Acknowledgment}

Financial assistance for this research by the NSF (CHE-0610349) and NIH (General Medical Sciences, R01 GM075107) is gratefully acknowledged.

\section{References}

(1). (a)Bogdanović HB, Meister B, Pauling H. Angew. Chem., Int. Ed. Engl 1972;11:1023.(b) For a recent review, see:RajanBabu TV. Chem. Rev 2003;103:2845. [PubMed: 12914483]

(2). Ni:(a)Nomura N, Jin J, Park H, RajanBabu TV. J. Am. Chem. Soc 1998;120:459.(b)RajanBabu TV, Nomura N, Jin J, Nandi M, Park H, Sun X. J. Org. Chem 2003;68:8431. [PubMed: 14575468]Ru: (c)He Z, Yi CS, Donaldson WA. Org. Lett 2003;5:1567. [PubMed: 12713325]Co:(d)Grutters MMP, Müller C, Vogt D. J. Am. Chem. Soc 2006;128:7414. [PubMed: 16756275]Pd:(e)Englert U, Haerter R, Vasen D, Salzer A, Eggeling EB, Vogt D. Organometallics 1999;18:4390.(f)Shi W-J, Xie J-H, Zhou Q-L. Tetrahedron: Asymmetry 2005;16:705.

(3). Zhang A, RajanBabu TV. Org. Lett 2004;6:1515. [PubMed: 15101781]

(4). For a review of synthesis of 2-arylpropionic acids, see:Stahly GP, Starrett RM. Collins AN, Sheldrake GN, Crosby J. Chirality in Industry II. 1997John WileyChichester

(5). (a) Feringa BL. Acc. Chem. Res 2000;33:346. [PubMed: 10891052] (b) Arnold LA, Imbos R, Mandoli A, de Vries AHM, Naasz R, Feringa BL. Tetrahedron 2000;56:2865.

(6). (a) Franció G, Faraone F, Leitner W. J. Am. Chem. Soc 2002;124:736. [PubMed: 11817933] (b) Kumareswaran R, Nandi N, RajanBabu TV. Org. Lett 2003;5:4345. [PubMed: 14601996] (c) Zhang 
A, RajanBabu TV. J. Am. Chem. Soc 2006;128:54. [PubMed: 16390118] (d) Zhang A: RajanBabu TV. J. Am. Chem. Soc 2006;128:5620. [PubMed: 16637613] (e) Shi W-J, Zhang Q, Xie J-H, Zhu S-F, Hou G-H, Zhou Q-L. J. Am. Chem. Soc 2006;128:2780. [PubMed: 16506739]

(7). For representative examples of finely tuned phosphoramidites from various research groups, see:(a) Alexakis A, Polet D, Rosset S, March S. J. Org. Chem 2004;69:5660. [PubMed: 15307737](b) Bernsmann H, van den Berg M, Hoen R, Minnaard AJ, Mehler G, Reetz MT, De Vries JG, Feringa BL. J. Org. Chem 2005;70:943. [PubMed: 15675853](c)Streiff S, Welter C, Schelwies M, Lipowsky G, Miller N, Helmchen G. Chem. Commun 2005:2957.(d)Leitner A, Shekhar S, Pouy MJ, Hartwig JF. J. Am. Chem. Soc 2005;127:15506. [PubMed: 16262414](e)Yu RT, Rovis T. J. Am. Chem. Soc 2006;128:12370. [PubMed: 16984159](f)Du H, Yuan W, Zhao B, Shi Y. J. Am. Chem. Soc 2007;129:11688. [PubMed: 17803307]

(8). See Supporting Information for experimental details and a more complete list of ligands.

(9). To the best of our knowledge, this ligand has not been described in the literature.

(10). For the best asymmetric routes to date, Naproxen via Ru-catalyzed asymmetric hydrogenation of 2-arylacrylic acids:(a)Ohta T, Takaya H, Kitamura M, Nagai K, Noyori R. J. Org. Chem 1987;52:3174.(98\% ee). Ni-catalyzed asymmetric hydrocyanation:(b)RajanBabu TV, Casalnuovo AL. J. Am. Chem. Soc 1996;118:6325. and references cited therein (95\% ee). Ibuprofen via Rucatalyzed hydrogenation:(c)Uemura T, Zhang X, Matsumura K, Sayo N, Kumobayashi H, Ohta T, Nozaki K, Takaya H. J. Org. Chem 1996;61:5510.(97\% ee). Rh-catalyzed asymmetric hydroformylation:(d)Nozaki K, Sakai N, Nanno T, Higashijima T, Mano S, Horiuchi T, Takaya H. J. Am. Chem. Soc 1997;119:4413.(92\% ee). Hydrovinylation: ref 3 (91\%). No useful catalytic asymmetric methods are known for other $(S)$-2-arylpropionic acid precursors.

(11). Except for Diels-Alder reactions, asymmetric catalyzed C-C bond-forming reactions of acyclic 1,3dienes give only moderate regio- and enantioselectivities. See, for example: (a)

cyclopropanation:Doyle M. Ojima I. Catalytic Asymmetric Synthesis. 2000Wiley-VCHNew York (b) ene reaction:Terada M, Mikami K. J. Chem. Soc., Chem. Commun 1995:2391.(c) Hydroformylation:Horiuchi T, Ohta T, Shirakawa E, Nozaki K, Takaya H. Tetrahedron 1997;53:7795.(d) Hydrocyanation:Saha B, RajanBabu TV. Org. Lett 2006;8:4657. [PubMed: 16986974]

(12). He Z, Yi CS, Donaldson WA. Synlett 2004:1312.

Org Lett. Author manuscript; available in PMC 2009 September 17. 
<smiles>CC(c1ccccc1)N(C(C)c1ccccc1)p1oc2ccc3c(c2c2c4c(ccc2o1)C=CI=C4)C=IC=C3</smiles><smiles>[X]N(C(C)c1ccccc1)p1oc2ccc3ccccc3c2c2c(ccc3ccccc32)o1</smiles><smiles>[Y]C(C)N([X])p1oc2ccc3ccccc3c2c2c(ccc3ccccc32)o1</smiles>

(rac)-L, $\mathbf{L}_{1}+\mathbf{L}_{2}(1: 1)$

$\mathbf{L}_{4}, R_{\mathrm{a}} S_{\mathrm{c}}, \mathrm{X}=$ methyl

$\mathrm{L}_{7}, R_{\mathrm{a}} R_{\mathrm{c}}, \mathrm{X}=$ benzyl, $\mathrm{Y}=$ phenyl

$L_{1}, S_{c} S_{c}$, biphenyl

$\mathrm{L}_{5}, R_{\mathrm{a}} R_{\mathrm{c}}, \mathrm{X}=$ isopropyl

$L_{8}, R_{\mathrm{a}} S_{\mathrm{c}}, \mathrm{X}=$ benzyl, $Y=$ phenyl

$\mathrm{L}_{2}, R_{\mathrm{c}} R_{\mathrm{c}}$, biphenyl

$L_{6}, R_{\mathrm{a}} S_{\mathrm{c}}, \mathrm{X}=$ isopropyl

$L_{9}, R_{a} S_{c}, X=1-n a p h t h y l m e t h y l, Y=$ phenyl

$L_{3}, R_{\mathrm{a}} S_{c} S_{c}$, binaphthyl

$L_{10}, R_{a} S_{c}, X=$ benzyl, $Y=1$-naphthyl

Figure 1.

Selected phosphoramidite ligands. 


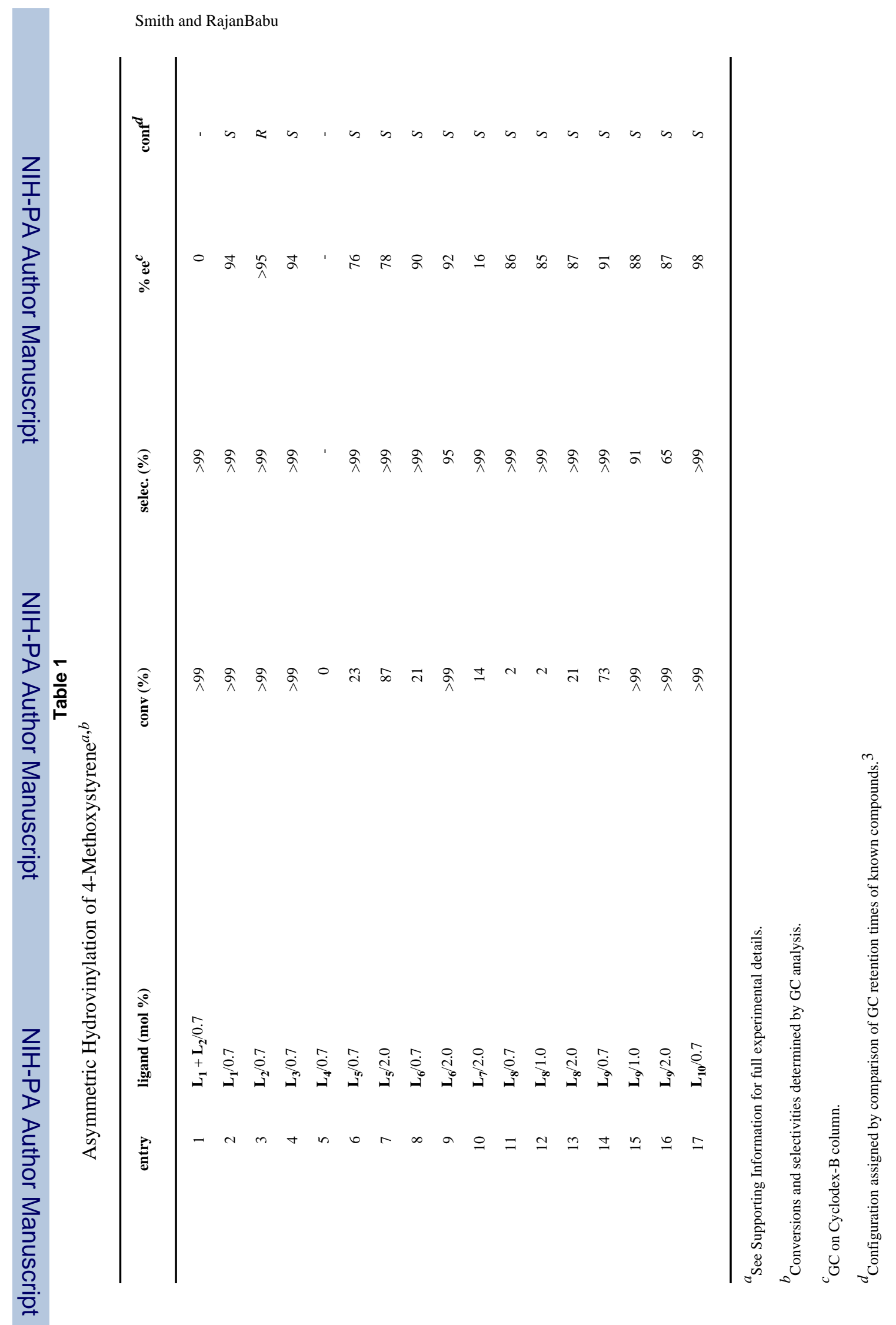

Page 6 


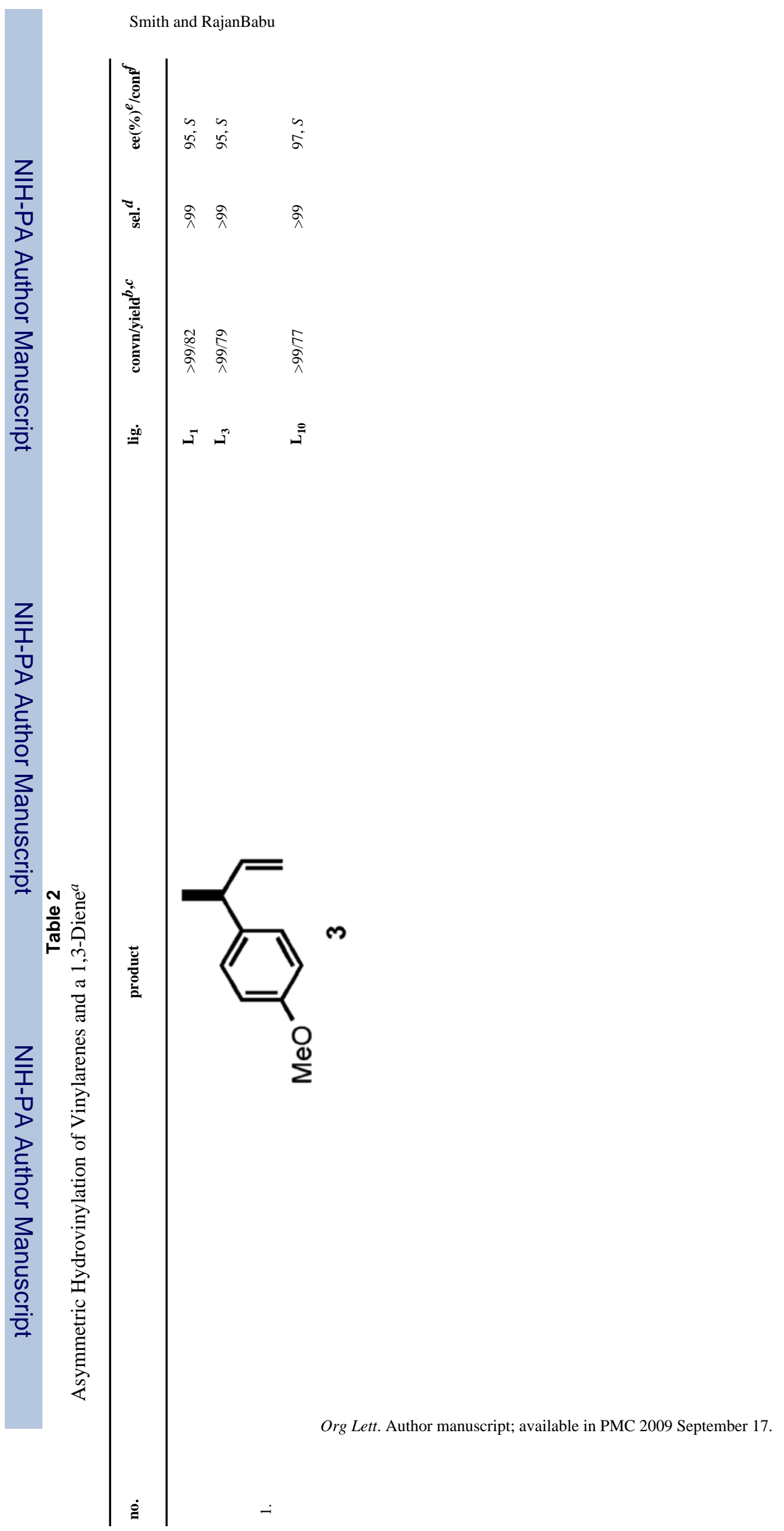




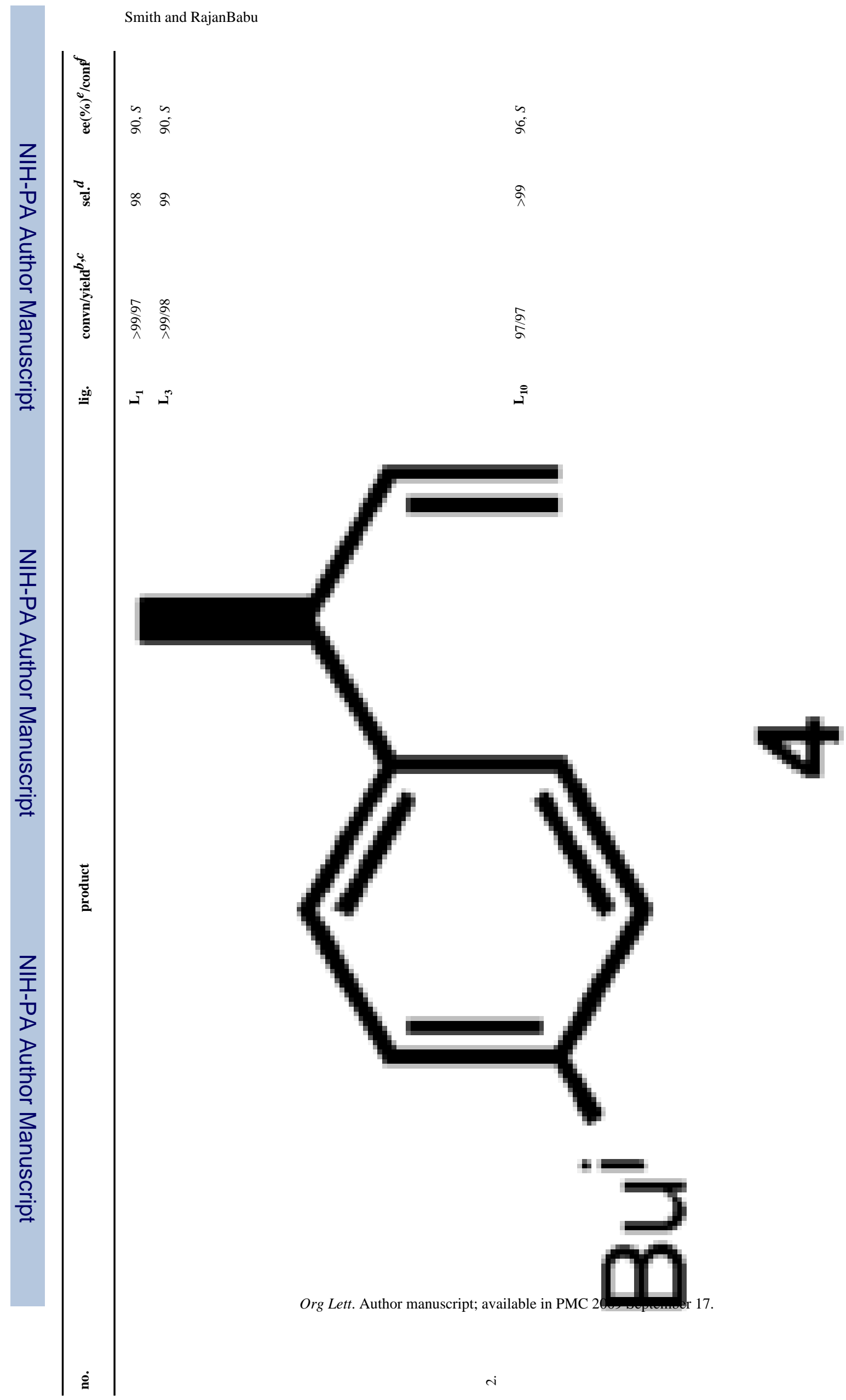




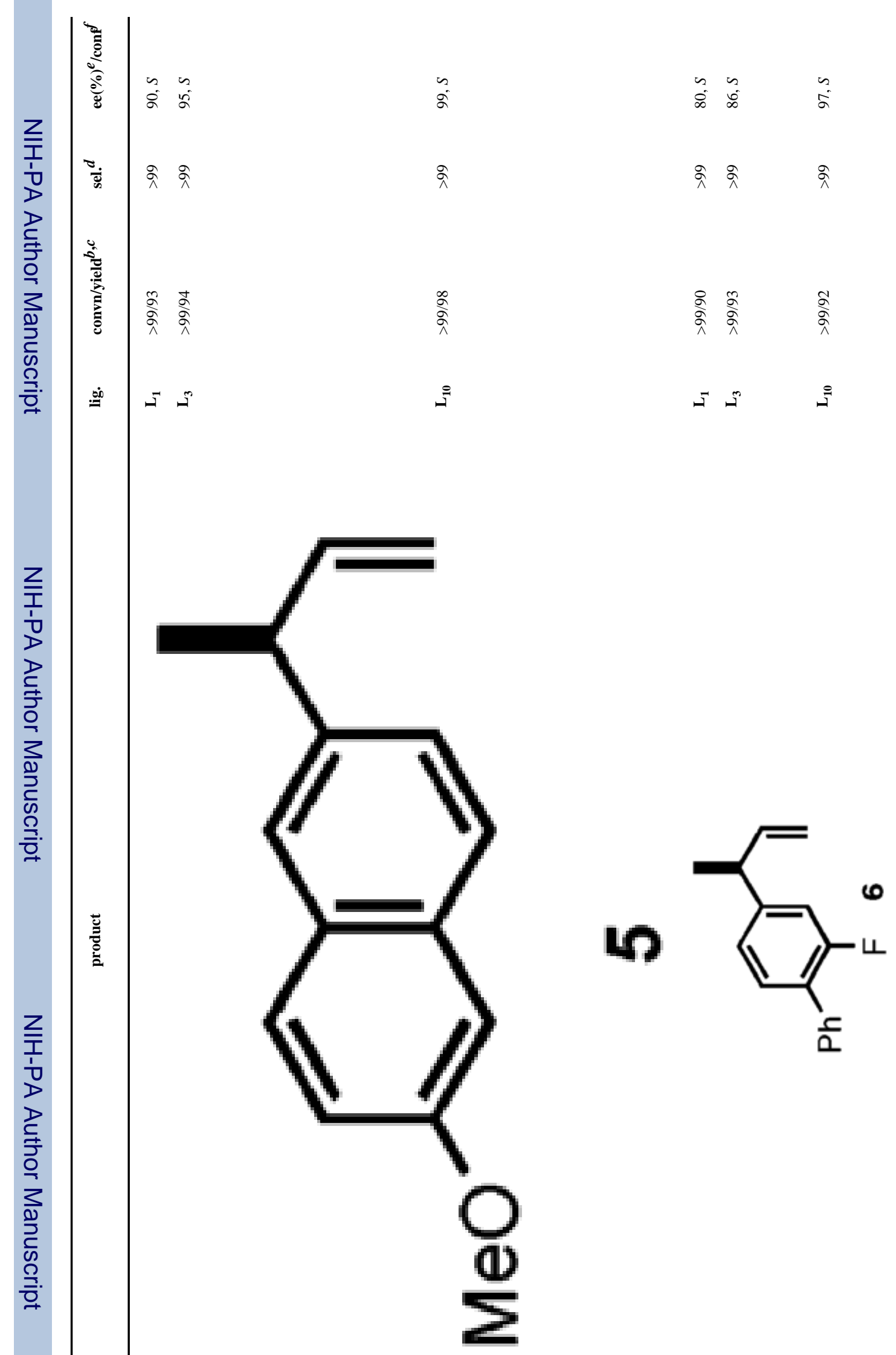

Org Lett. Author manuscript; available in PMC 2009 September 17. 


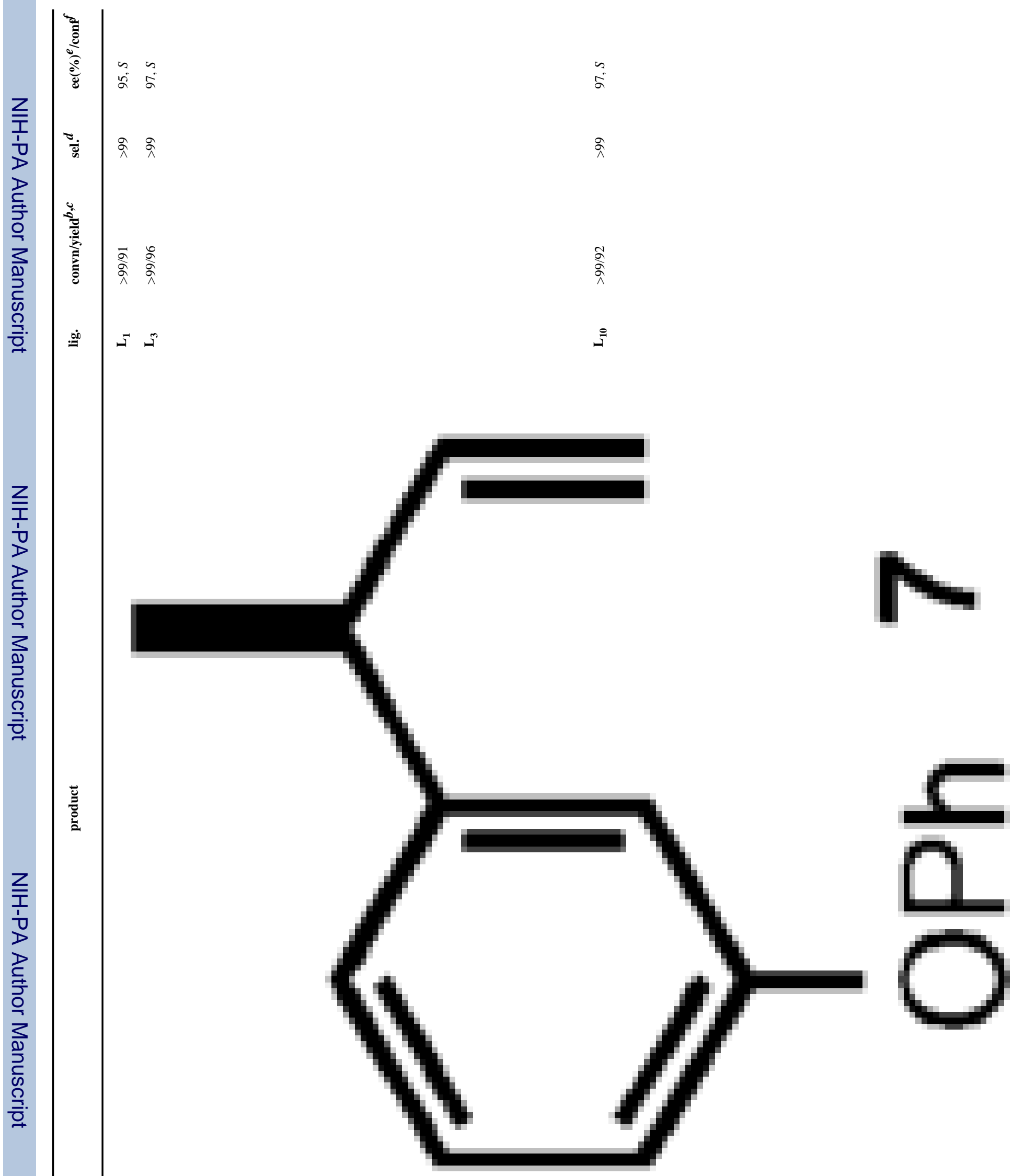

Org Lett. Author manuscript; available in PMC 2009 September 17. 

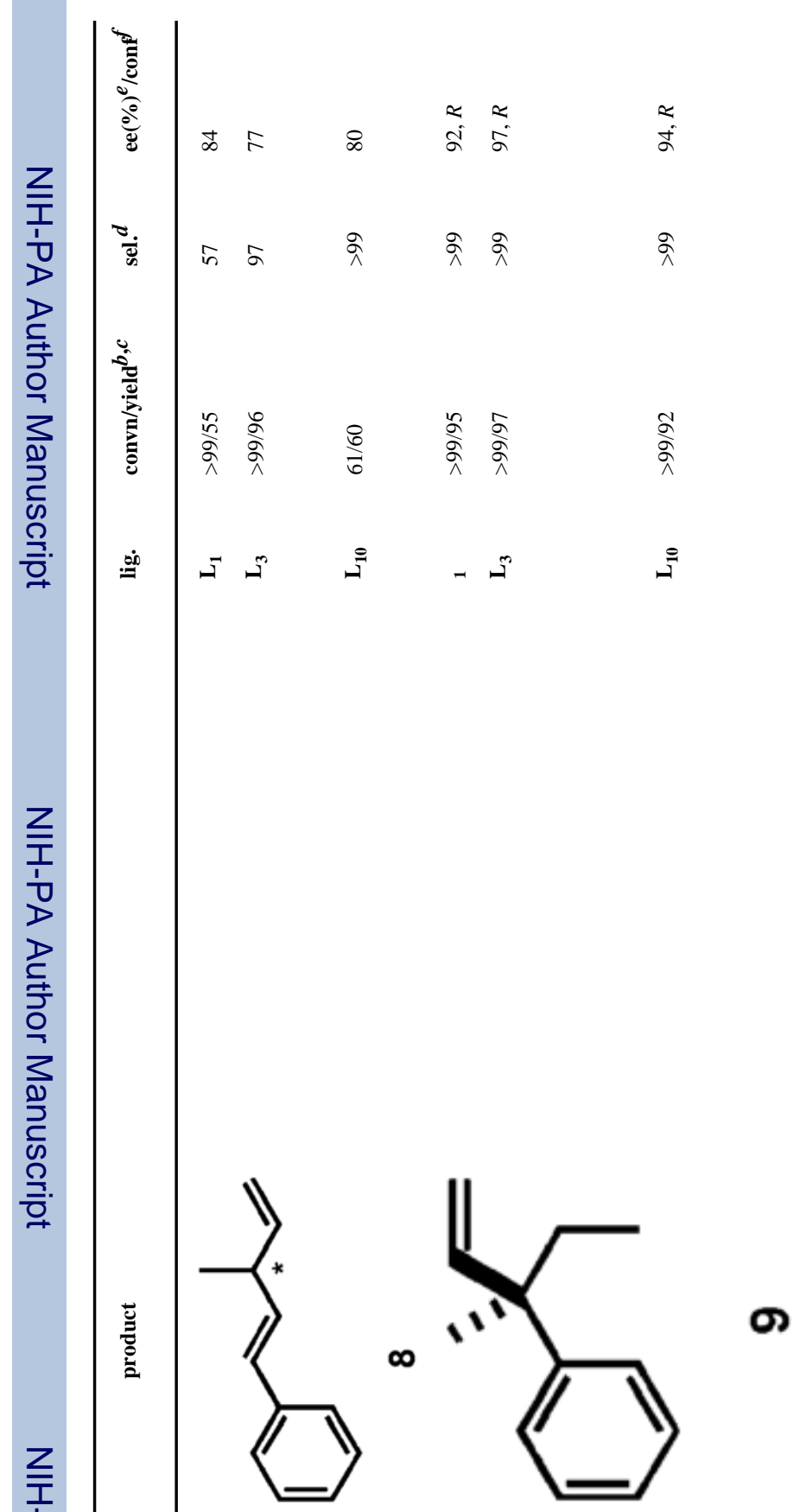

Org Lett. Author manuscript; available in PMC 2009 September 17. 


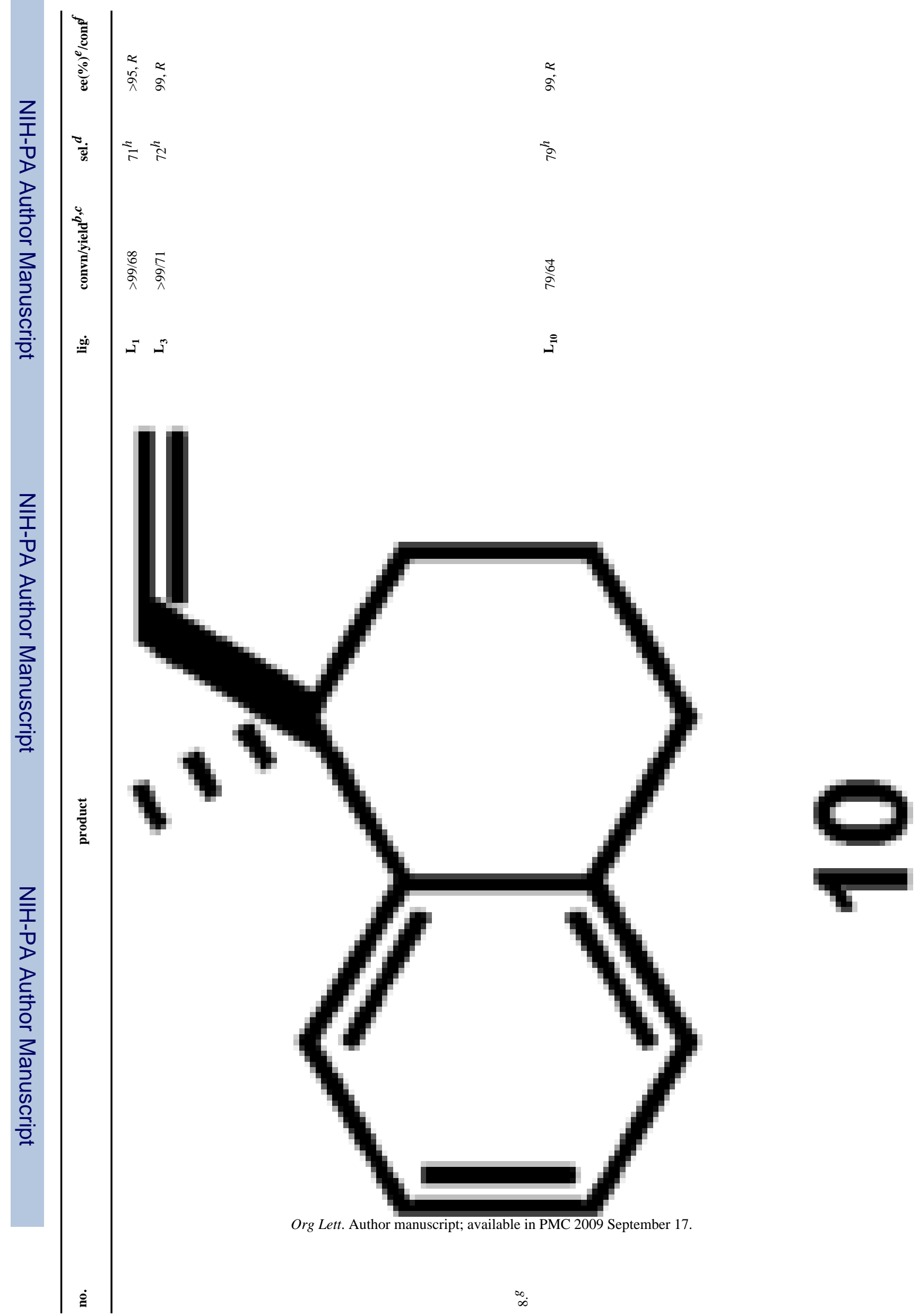




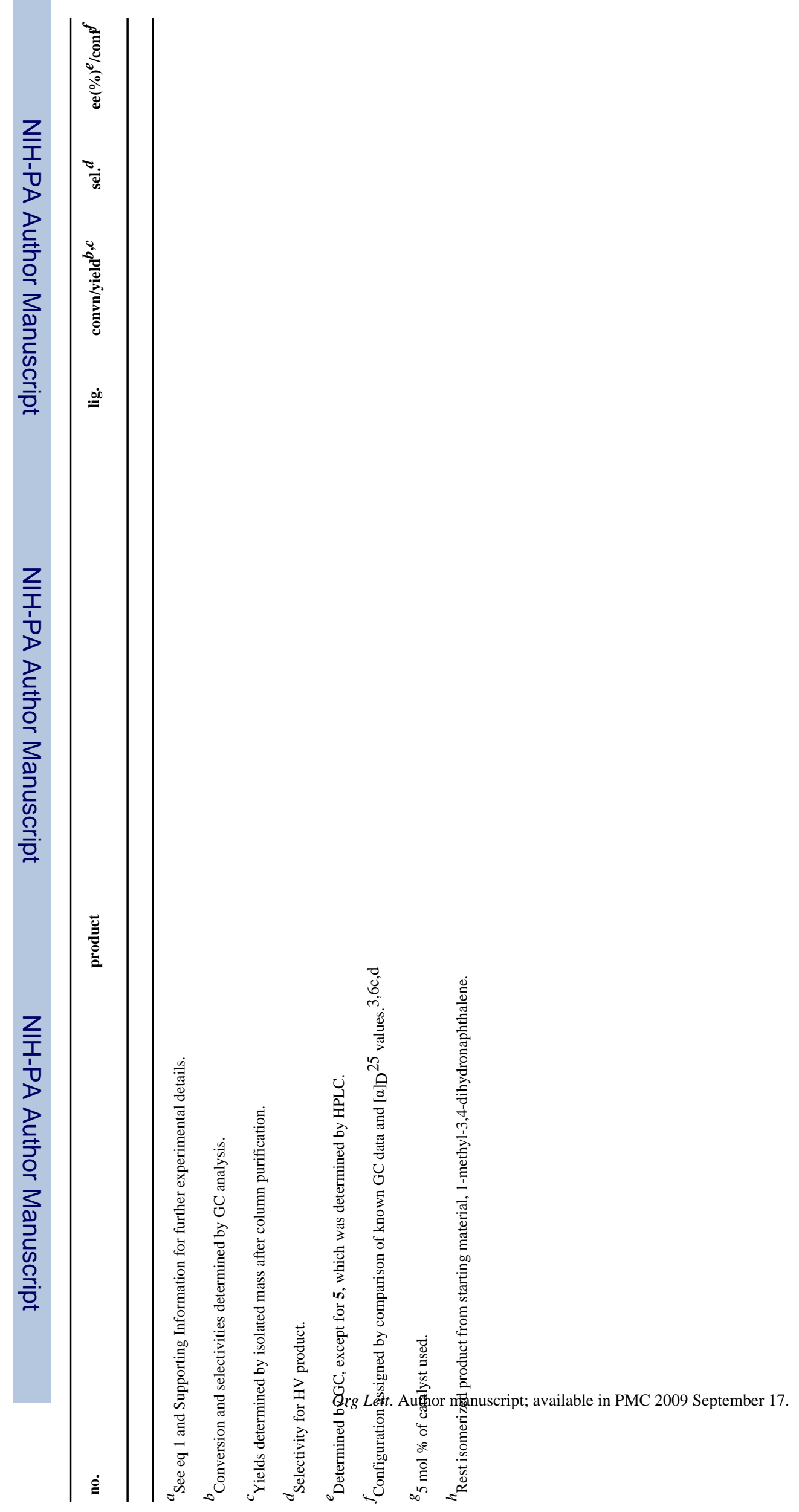

